\title{
Hacia una (co)educación sexual inclusiva. Aportes desde la investigación sobre infancia y juventud trans
}

\author{
Luis Puche* \\ Universidad de Málaga
}

\author{
PALABRAS CLAVE \\ Educación sexual \\ Diversidad trans \\ Discriminación \\ Coeducación \\ Inclusión
}

\begin{abstract}
RESUMEN
A partir de una investigación etnográfica desarrollada en España entre los años 2013 y 2018, en este artículo se exploran las experiencias y perspectivas de personas jóvenes que se identifican como trans (transexuales, transgénero) y de sus familias en relación con la sexualidad, la afectividad y la educación. Sus vivencias están marcadas por la escasez de referentes y sometidas a distintas formas de violencia y discriminación que nacen del sistema sexo/género; al mismo tiempo, están generando innovaciones emancipatorias a nivel individual y colectivo. El papel de la educación sexual en las trayectorias escolares de los y las jóvenes trans aparece descrito como deficitario debido al carácter puntual de estas acciones formativas (cuando se dan) y a su escaso énfasis en la diversidad. Se concluye afirmando la necesidad de una educación sexual formalizada que resulte inclusiva para todas las personas, en un marco coeducativo y de cultura de paz.
\end{abstract}

\section{Towards an inclusive sex (co)education. Contributions of a research on trans childhood and youth}

\begin{abstract}
Based on an ethnographic research carried out in Spain between 2013 and 2018, this article explores the experiences and perspectives of young people who identify themselves as trans (transsexuals, transgender) and their families in relation to sexuality, affectivity and education. These experiences are marked by the scarcity of models and are subjected to different forms of violence but, at the same time, are generating emancipatory innovations at the individual and collective level. Attention is also paid to the deficient role of sex education in the lives of trans youth, to the punctual nature of these training actions (when they exist) and to their scant emphasis on diversity. The article concludes by affirming the need for a formalized sexual education that is inclusive for all people, in a coeducational framework and a culture of peace.
\end{abstract}

Universidad de Málaga

Autor de correspondencia: * Luis Puche; luis.puche@uma.es

Recibido: 31/05/2021 - Aceptado: 15/09/2021

Revista de Formación del Profesorado e Investigación Educativa

Facultad de Formación del Profesorado y Educación

Universidad de Oviedo

Enero - Diciembre 2021

ISSN: $2340-4728$

(c) (1) $\circledast)$ Esta obra está bajo una licencia internacional Creative Commons

Esta obra está bajo una licencia internacional
Atribución-NoComercial-SinDerivadas 4.0. 


\section{Introducción}

Tanto la coeducación como la educación sexual cuentan en el Estado Español con dilatadas trayectorias y han sido objeto de complejos avatares intelectuales, históricos y legislativos (Ballarín, 2019; Calvo, 2021; Hurtado y Terrón, 2020;). Sin embargo, su implementación formalizada e integral en el sistema educativo español plantea aún importantes carencias y retos. La presencia efectiva de estos contenidos y enfoques pedagógicos en nuestros centros escolares sigue dependiendo en gran medida de la voluntad y el compromiso del profesorado o de los centros a título individual. En el caso de la coeducación -entendida esta no solo como la educación de chicos y chicas por igual, sino como una educación no sexista encaminada a erradicar las fuentes de la desigualdad y generar una conciencia crítica respecto de ella-, señala Ballarín que, a pesar de los avances logrados, el androcentrismo persiste y se hacen necesarias "nuevas medidas políticas que sean taxativas para convertir el voluntarismo de algunas y algunos en obligación de todos y todas y formarse en los conocimientos acumulados por el feminismo académico" (Ballarín, 2019: 197). Calvo, por su parte, subraya igualmente en relación con la educación sexual la falta de formación inicial del profesorado y la carencia de "espacios formales en el currículo educativo destinados a incorporar la educación sexual como cuerpo teórico propio" (Calvo, 2021: 298).

No podemos perder de vista, por otra parte, que en la actualidad los cambios sociosexuales se suceden a gran velocidad (Plummer, 2011: xviii-xix) y que están emergiendo entre la población más joven nuevas formas afirmativas de habitar el cuerpo, el género, la sexualidad y el deseo que en muchos casos desafían las dicotomías tradicionales (masculino/femenino, heterosexualidad/ homosexualidad) y que, de manera creciente, se reivindican como feministas. Al mismo tiempo que esto ocurre, observamos también movimientos en sentido contrario: hacia la perpetuación o el reforzamiento de ciertos estereotipos discriminatorios y dinámicas sexistas en la construcción de la personalidad y en las relaciones interpersonales, particularmente las amorosas, y en los modelos estéticos, comportamentales o sexuales juveniles (León y Aizpurúa, 2020; Pibernat, 2021). Todo ello se encuentra además vehiculado por los mass media y las nuevas tecnologías contenidas en el smartphone, que están facilitando conexiones y redes comunitarias al tiempo que engendran nuevas formas de violencia (Benítez, 2016). En este marco complejo y cada vez más plural, se hace urgente seguir pensando y adecuando a los tiempos veloces en que vivimos las nutridas tradiciones y bagajes de lo que aquí denominaremos de manera sincrética (co)educación sexual, con la ambición de poner en sintonía las diversidades emergentes con el principio universalista de igualdad.

Queriendo contribuir a este proceso, el presente artículo se plantea como objetivo principal aprender de las experiencias y perspectivas de una parte pequeña, aunque cada vez más visible, de la población joven: las personas que se identifican como trans (transexuales, transgénero). Para ello, se parte de una investigación doctoral (Puche, 2018) en la que se indagó sobre la emergencia en el contexto español de una nueva categoría social, la de la infancia y la juventud trans, y sobre las contradicciones, procesos de cambio y desafíos a los que ha conducido en los terrenos educativo, legislativo, comunitario, familiar y de la producción de nuevas subjetividades en un marco de desigualdad estructural.

\section{Realidades trans y sistema sexo/género}

Es cada vez más habitual que se utilice la fórmula "personas trans" como término paraguas para referirse a aquellas personas cuya identificación subjetiva y su manera de presentarse ante el mundo no coinciden con la asignación de sexo/género que han recibido en el momento de nacer en base a su anatomía genital. A partir de este elemento común, los itinerarios de vida son diversos y pueden incluir en distinto grado (o no incluirlas) modificaciones corporales e intervenciones médicas (hormonales, quirúrgicas) y cosméticas para adecuar la apariencia física al sexo/género reivindicado como propio. Dependiendo de ello y de las autodenominaciones individuales y colectivas (que varían en función del contexto geográfico) podemos hablar de transexualidad, transgenerismo, realidades trans $\mathrm{u}$ otras categorías de reciente aparición (Platero, 2014; Stryker, 2008;).

La más conocida y usada de estas categorías ha sido, durante largo tiempo, la noción de "transexualidad" que, sin embargo, está siendo sometida a crítica en las últimas décadas por sus connotaciones médicas y por llevarnos a pensar de forma automática en itinerarios quirúrgicos y en procesos de normalización de género según un esquema dual. La universalidad de esta categoría ha sido puesta en cuestión desde los estudios antropológicos realizados en sociedades no occidentales (Nieto, 2008). Además, la crítica y las propuestas alternativas han provenido también del caudal de teoría, conocimiento académico, innovaciones personales y discurso político que muchas personas trans han desarrollado bajo el paradigma del "transgenerismo" (Feinberg, 1992), así como desde la lucha por la despatologización de la transexualidad (Araneta, 2013; Suess, 2018). Como resultado provisional de este proceso de reflexión categorial y vital, se ha acuñado de forma más reciente la fórmula "personas trans" que empleamos en este artículo como un término abarcador, no necesariamente vinculado a la medicalización y de amplio consenso (Platero, 2014: 23).

La aplicación de estas categorías a la infancia y la juventud es una novedad muy reciente en el contexto español y su análisis nos permite, al menos, dos operaciones simultáneas. Por una parte, arrojar luz sobre un fenómeno social minoritario, de enorme complejidad, que está atravesado de potencialidades transformadoras pero también de injusticias, dificultades y riesgos relacionados con la patologización, la medicalización y las violencias sociales que a menudo acompañan a las vivencias trans. Por otra parte, nos ofrece un lugar privilegiado desde el que mirar hacia los procesos de socialización de género que afectan a toda la sociedad. En ese sentido, las narrativas de las personas trans, la calidad de sus experiencias vitales, sus aportaciones e innovaciones sociales y las violencias escolares, familiares y comunitarias que a menudo padecen nos hablan de los niveles de igualdad o desigualdad entre hombres y mujeres que existen en un determinado contexto y del grado de vigencia del sexismo, de la transfobia y la homofobia; es decir, del funcionamiento del sistema sexo/género.

Desde la antropología, se ha conceptualizado como "sistema sexo/género" o "sistema de género" (Rubin, 1986) al entramado multidimensional -y variable dependiendo del contexto cultural e histórico- que regula socialmente el género y la sexualidad: ese lugar social en el que la materia biológica del sexo es convertida por las relaciones sociales de desigualdad en un sistema de prohibiciones, obligaciones y derechos diferenciales para unos y otros individuos (Maquieira, 2005: 162). Se utiliza también el término "patriarcado" para poner el énfasis crítico en la dominación masculina sistémica y en la opresión de las mujeres y de las personas que rompen con el orden de género tanto en nuestra sociedad como en otras. De acuerdo con Barragán-Medero y Pérez-Jorge (2020: 2), "la organización patriarcal incluye el género como una forma de dividir a las personas y perpetuar la opresión" al tiempo que refuerza la heterosexualidad como modelo hegemónico excluyente -basado en la complementariedad de los sexos-e impide la autonomía en la toma de decisiones sobre nuestras preferencias sexuales. Es lo que Butler (2015) ha conceptualizado, por su parte, como "matriz heterosexual". Sexo, género y sexualidad aparecen, por tanto, como entidades socialmente interdependientes y arraigadas en un mismo sistema o matriz sociocultural.

Desde esta perspectiva sistémica, el sexismo, la homofobia, la transfobia y la LGBTIfobia (según la fórmula recientemente acuñada) no pueden ser entendidos como actitudes o sentimientos 
individuales, sino como prejuicios discriminatorios construidos culturalmente e interiorizados a través de la socialización (Pichardo, 2015:12). Centrándonos ya específicamente en la homofobia y la transfobia, resulta útil distinguir entre la una y la otra a la hora de establecer sus especificidades, aunque al nacer del mismo sistema social las fronteras entre ellas son difusas. El término homofobia se refiere a la opresión contra aquellas personas en las que se percibe atracción sexual hacia las personas de su mismo sexo (al margen de cualquier consideración en cuanto a su identidad o expresión de género). La transfobia, por su parte, alude a la penalización de la falta de correspondencia entre sexo y género, así como de las expresiones de género no convencionales. Ambas actúan sobre toda la población y no únicamente sobre las personas trans, gais o lesbianas, cimentando el orden sexual y las fronteras entre los géneros y mostrando "la ferocidad con que la sociedad defiende la vigencia de sus sistemas clasificatorios" (Juliano, 2017: 30). Así lo explican Coll-Planas et al. (2009: 19):

La causa de la aversión hacia gais y lesbianas es que, al romper con la norma de la complementariedad hombre/mujer, alteran las reglas del género. Por tanto, la transfobia -la vigilancia de la correspondencia sexo/género- resulta más extendida y está más directamente relacionada con el mantenimiento del sexismo que la homofobia, que regula la elección de la pareja sexual. De hecho, podemos considerar que la homofobia acaba siendo una de las expresiones de la transfobia, en el sentido de que una forma de atacar a alguien que no actúa según el género normativo (un chico afeminado o una chica masculina) es tildarlo de gay o de lesbiana - utilizando a menudo palabras más despectivas.

Las discriminaciones que venimos abordando se transmiten no solo a través de mensajes o castigos directos sino, sobre todo, por medio de sistemas de endoculturación y conformidad de las voluntades, es decir, a través de los aprendizajes informales que vamos adquiriendo a lo largo de la vida por interiorización de los modelos dominantes. El sistema educativo y otras instituciones sociales (el derecho, los medios de comunicación, el sistema médico, el sistema de parentesco, entre otras), de una forma más estructurada, transmiten también una determinada cosmovisión y un cuerpo de conocimientos sobre lo que se considera normal, cierto, justo, saludable, significativo y pensable. En contextos de desigualdad, "ambas formas de presión (la informal y la formal) constriñen las capacidades individuales y presionan para realizar adaptaciones conductuales duras" (Juliano, 2004: 67).

Los entornos escolares son nodos importantes en este entramado sistémico. Es habitual que, en ellos, la transfobia y la homofobia se entrelacen cotidianamente con el sexismo (y con otros vectores de discriminación) dando lugar a procesos de acoso escolar entre los pares que condicionan fuertemente la construcción de la personalidad, de las relaciones de género y de la sexualidad en estas edades decisivas. Con el agravante de que muchas veces estas situaciones son institucionalmente silenciadas o ignoradas, trasladando a las víctimas del acoso la responsabilidad de mejorar su situación (Benítez, 2016; Elipe et al., 2017; Gallardo y Espinosa, 2021; Pichardo y de Stéfano, 2015; Richard y Chamberland, 2014; Takács, 2006; Saleiro, 2017).

A pesar de su capacidad constrictiva, el sistema sexo/género es un producto social y, como tal, es susceptible de ser cambiado a través de la acción humana. De hecho, en las últimas décadas el impacto del feminismo y de las luchas por las libertades sexuales no han cesado de producir transformaciones profundas en él. De ahí la importancia de reforzar los contenidos pedagógicos transformadores y de fomentar culturas escolares (y sexuales) que contribuyan a la erradicación de las violencias y al logro de la paz y la igualdad en un contexto de creciente diversidad. Para esta tarea contamos ya con un importante corpus de iniciativas y herramientas pedagógicas tanto a nivel nacional como in- ternacional (Barragán-Medero y Pérez-Jorge, 2020; Meyer, 2009; Pichardo, 2015; Platero, 2014; Sánchez et al., 2016; Sanchís, 2021; entre muchas otras).

\section{Objetivos}

Este artículo está orientado por tres objetivos:

- Dar cuenta de las vivencias en relación con el cuerpo, la sexualidad y la afectividad de las personas trans jóvenes, ubicándolas en el marco de un sistema sexo/género que produce desigualdades y exclusiones pero que también está sometido a cambios.

- Explorar el modo en el que se aborda la sexualidad en los entornos educativos formales desde la perspectiva de la juventud trans.

- Formular algunas claves y principios para una educación sexual inclusiva y coeducativa.

\section{Metodología}

Con el fin de satisfacer tales objetivos, se analiza una parte de los resultados de la tesis doctoral titulada "Infancias y juventudes trans. Una aproximación desde la Antropología Social", desarrollada en la Universidad Autónoma de Madrid y que implicó un trabajo de campo extensivo (entre 2013 y 2018) en distintos puntos de la geografía española, en contextos tanto rurales como urbanos. En el curso de la investigación se utilizaron diferentes técnicas de producción de datos, tanto cualitativas como cuantitativas, desde un enfoque etnográfico: entrevistas en profundidad, observación participante en entornos educativos y cuestionarios. En lo que respecta a las entrevistas, que son las que adquieren más importancia de cara a la elaboración de este artículo, se realizaron 42: 16 de ellas a familiares (mayoritariamente madres) de niñas y niños identificados como trans; 22 entrevistas a jóvenes a partir de 16 años y 4 entrevistas a profesorado y activistas. Por medio de ellas se pudo tener acceso, en total, a 33 casos de niños, niñas y jóvenes trans (19 se identificaban como mujeres y 14 como varones). El criterio de selección muestral estuvo orientado a explorar las experiencias de vida de personas que se definían o que eran definidas por sus entornos como transexuales o trans, por lo que la muestra no incluyó otros aspectos novedosos de la diversidad sexogenérica (personas no-binarias, queer, agénero, etc.).

Al tratarse de una investigación de carácter etnográfico, el objetivo no era lograr una muestra de informantes estadísticamente representativa; lo que sí que se buscó fue la heterogeneidad con el fin de analizar experiencias vitales en distintos contextos. Para ello, se estableció una estrategia de captación múltiple: correo de difusión en asociaciones y entidades comunitarias; captación durante las sesiones de observación-participante en establecimientos educativos y asociativos; contactos provenientes de las redes profesionales y de la actividad como formador del propio investigador; y bola de nieve. Se realizaron entrevistas en 7 comunidades autónomas españolas. El mayor número de informantes se ubicó en la Comunidad Canaria, gracias al apoyo de colaboradores locales del ámbito sanitario y asociativo. En Madrid, Andalucía, Aragón, Castilla y León, País Vasco y Castilla la Mancha se concentran, en orden decreciente, el resto de los casos estudiados. 16 de los casos se ubican en ciudades pequeñas y medianas; 12 casos corresponden a grandes ciudades (de más de 500.000 habitantes); y 5 casos, por último, provienen de poblaciones pequeñas del ámbito rural.

Para acceder a las narrativas de todas estas personas se hizo uso de la entrevista etnográfica: orientada por un guion temático que contenía unidades de análisis definidas de antemano, pero al mismo tiempo abierta y flexible. De ese modo, se buscó obtener relatos biográficos más que un encadenamiento de preguntas y respuestas (Del Olmo, 2003: 213-214). En todas las entrevistas se procuró una posición de "simetría ética" (Christensen y Prout, 2002) 
según la cual se ofrecía el mismo trato a las personas informantes independientemente de su edad, ya fueran adultas o jóvenes: explicando quién era el investigador, informando de los objetivos del estudio, garantizando el anonimato, recabando su consentimiento informado y animándolas a parar la entrevista o apagar la grabadora cuando lo estimasen oportuno. No se hicieron distinciones por edad en la forma de abordar las entrevistas más allá de adaptar el lenguaje cuando esto era necesario, mostrando siempre interés por sus experiencias, valores y perspectivas.

A lo largo del proceso etnográfico, se fueron definiendo una serie de unidades de análisis con una función múltiple: han servido para articular el diseño de los guiones de entrevista y observación, para orientar la redacción de las notas de campo y, finalmente, para analizar e interpretar todo el material discursivo y etnográfico producido (íntegramente transcrito). En esta última fase, las unidades fueron codificadas con ayuda del software de análisis de datos cualitativos Atlas.ti (Trinidad et al., 2006). En definitiva, el proceso de elaboración de categorías analíticas combinó una fase de categorización deductiva inicial con la emergencia dinámica de categorías inductivas provenientes del propio material etnográfico.

Es preciso señalar, por último, que en la escritura de este texto se ha respetado el género reivindicado por cada una de las personas que aparecen representadas en él. Así, cuando se habla de chicas trans, se trata de personas que fueron consideradas varones al nacer en base a su anatomía genital pero que se identifican como mujeres y en femenino. Cuando se habla de chicos trans, se trata de personas asignadas como mujeres al nacer pero que se viven como hombres y en masculino. Con objeto de proteger su anonimato, todos los nombres de informantes que se citan en el texto son ficticios.

\section{Resultados. Sexualidades y afectividades en los márgenes de la escuela}

\section{Vivencias, violencias, búsquedas, innovaciones}

Las personas trans y, en general, aquellas que rompen con las normas de género en la infancia y la juventud son especialmente vulnerables frente a la presión coercitiva para que se ajusten a la norma. Es frecuente que sufran rechazo por parte de miembros de su familia, que afronten correcciones constantes respecto de sus comportamientos e identificaciones de género y que sean víctimas de acoso escolar y estigmatización entre sus pares. Estas violencias las reciben en mayor medida las chicas trans de acuerdo con una lógica de "transmisoginia" (Serrano, 2007) que es coherente con un sistema de género patriarcal en el que pasar a ser mujer supone un descenso en la escala social. Todo ello provoca elevados riesgos de aislamiento social, de que se les diagnostiquen trastornos del aprendizaje o de desarrollar problemas psicológicos causados por el estrés, así como depresión e ideaciones suicidas (Travers, 2014). Tales presiones se traducen también en procesos de rechazo al propio cuerpo y en la necesidad psicológica y somática de emprender itinerarios corporales medicalizados que permitan la conciliación entre la autopercepción y la mirada de los otros. Las palabras de Ágata (16 años) resultan ilustrativas: "Yo antes me pasaba todas las noches llorando (...) La psiquiatra me tuvo que mandar una pastilla a consecuencia del [acoso que sufría en el] instituto y porque tenía un pequeño trauma al futuro: tenía miedo a no tener hijos, a no operarme, a no tener pareja".

Es habitual encontrar en sus narrativas la convicción de que no podrán ser personas deseadas en el plano sexual y/o afectivo. Tal y como lo expresaba también Lydia (21 años): "Yo pensaba que al hacer esto me iba a quedar sola de por vida (...) [Me decía a mí misma:] olvídate de que se te acerque nadie, sea mujer, hombre o perro". Otras informantes explicaban que recibían insultos por parte de determinados varones cuando estos estaban en pandilla, pero que esos mismos chicos les hacían proposiciones sexuales en priva- do (a través de las redes sociales virtuales). Esta lógica de atracción privada y desprecio público podemos conectarla con los patrones de construcción de la masculinidad en un marco heteronormativo: ciertos varones las desean como compañeras sexuales pero siempre a condición de que ello no salga a la luz pública, temerosos de la devaluación social que podrían experimentar al ser relacionados con mujeres trans (un riesgo que algunas chicas describen como miedo a la "homosexualización"). La deseabilidad sexual aparece, además, desconectada de la deseabilidad afectiva y ello supone un obstáculo importante para el reconocimiento generalizado, abierto, de las personas trans (particularmente de las mujeres) como sujetos deseados, sexual y afectivamente. No se desprende lo mismo del discurso de los chicos trans, que relataban haber vivido relaciones sexoafectivas satisfactorias con mucha mayor frecuencia. En la mayoría de los casos se trataba de relaciones mantenidas con chicas, cuya socialización no está tan lastrada por el miedo al contagio del estigma homosexual como la de los varones.

Los entornos virtuales son percibidos como lugares de riesgo por parte de algunas informantes, de nuevo especialmente por parte de ellas: algunas sospechan sistemáticamente de las muestras de interés afectivo sexual que reciben en las redes porque las consideran señuelos para hacerlas caer en una trampa. Este miedo a la transfobia en el ciberespacio no carece de fundamento. Candela (16 años), por ejemplo, narraba que se habían publicado fotos de ella en algunas redes sociales acompañadas de comentarios ofensivos y discriminatorios por parte de sus compañeros de instituto. La vida virtual que cada vez ocupa más horas del día entre los y las jóvenes favorece procesos de distanciamiento, desconexión moral y de cosificación violenta del otro al abrigo del anonimato (lo que se materializa con frecuencia en procesos de ciberacoso). Paradójicamente, la vida online también hace posible una sociabilidad alternativa que en ocasiones ayuda a sortear las dificultades que se dan en el entorno presencial. No en vano, varias de las chicas y chicos entrevistados han conocido allí a amistades y parejas.

A pesar de que estas dificultades (virtuales y presenciales) tienen un peso considerable en las vidas de muchas personas trans, cuando nos aproximamos a las historias afectivas y sexuales de algunas de ellas nos encontramos también con formas novedosas y emancipadoras de habitar la sexualidad, la corporalidad y el deseo. En este sentido, suele resultar clave el haber encontrado en algún momento de la biografía sexoafectiva a chicos o chicas que han decidido involucrarse con ellas en relaciones no solo sexuales sino también de pareja. Como se ha desarrollado más extensamente en otro lugar (Puche, 2021), estas relaciones tienen un impacto muy positivo en sus procesos de autoestima corporal e incluso en la aceptación de su anatomía de origen.

Señala Bozon (2013: 89), que los deseos y las relaciones sexuales necesitan de una implicación por parte de los actores que los ponen en práctica "y de improvisaciones personales e interpersonales complejas que se elaboran a partir de experiencias vividas y de representaciones culturales". En el caso de las sexualidades trans, este nivel de las improvisaciones personales e interpersonales cobra una importancia fundamental debido a que apenas existen referentes ni imaginarios o modelos que permitan a las personas trans jóvenes construir sus relaciones. De ahí que sean recurrentes las narraciones biográficas que nos hablan de una búsqueda, de un ir descubriendo y experimentando con prácticas y formas de aproximación sexual casi siempre intuitivas y que involucran un gran componente de vulnerabilidad.

En estos procesos de búsqueda, inciden muy positivamente las relaciones de pareja, pero también la información divulgada en Internet, las comunidades virtuales, el acompañamiento de ciertos profesionales y los grupos de autoapoyo de jóvenes trans que se organizan desde algunas asociaciones o centros de salud, en los que se intercambian experiencias y en cuyo seno se tiene la oportunidad de ampliar los horizontes de lo posible en el plano corporal y sexual desde el reconocimiento mutuo. Fue en uno 
de estos grupos de varones trans donde Arturo (23 años) decidió plantear ante el resto de sus compañeros su bisexualidad recién descubierta, que le ha provocado más de un quebradero de cabeza al no contar con referentes al respecto. La pluralidad de orientaciones sexuales y su independencia con respecto del hecho de ser o no trans han estado presentes entre las y los informantes de esta investigación, así como las relaciones afectivas y sexuales mantenidas entre personas trans (chica/chico, chica/chica, chico/ chico), que tampoco son infrecuentes.

En lo que atañe a la infancia, es reseñable la puesta en circulación de un imaginario corporal transgénero (la posibilidad de que existan "niños con vulva" y "niñas con pene") por parte del movimiento asociativo de las familias de "menores trans" (Asociación Chrysallis Euskal Herria, 2016). En cierto sentido, estaríamos ante una ruptura parcial de las retóricas del "cuerpo equivocado" -la idea de que las personas trans han nacido en cuerpos que nos les pertenecen, que son un error (Missé, 2018)- que han acompañado tradicionalmente a la transexualidad. Algunas familias, además, están llevando a cabo prácticas de crianza atentas a la estima corporal de sus hijos e hijas; el objetivo es conciliar la identificación de género trans con la aceptación de la anatomía y la fisiología de nacimiento, abriendo la posibilidad para que la cirugía genital no sea una necesidad vivida en la edad adulta (aunque la hormonación suele ser vista como algo inevitable). Es de esperar, en consecuencia, que las generaciones de personas trans que ahora se encuentran en la infancia o la juventud, desarrollen biografías corporales distintas a las que hasta ahora hemos conocido en las personas trans de otras generaciones.

Otro elemento de cambio que se ha captado en esta investigación tiene que ver con las posiciones críticas de algunos chicos y chicas trans, así como de sus familias, con respecto al modelo medicalizado de transexualidad y sus implicaciones corporales. La medicalización, en estos casos, aparece como una estrategia consciente, voluntaria e informada que se emprende tras la evaluación detenida de sus beneficios psicosociales y de sus inconvenientes en el curso de una toma de decisiones que comporta dilemas complejos. Y ello lleva a itinerarios de vida que no siempre son unidireccionales, sino que admiten matices, vueltas atrás, renegociaciones y modulaciones de los procesos de hormonación y de los cambios corporales.

La incorporación del feminismo por parte de la población más joven, por otro lado, también está favoreciendo posturas críticas y una toma de distancia con respecto a la masculinidad hegemónica (Connell, 1995), incluida aquella encarnada por varones trans: “ $\mathrm{Ni}$ coincido con el estereotipo masculino ni tengo la intención ni me identifico con él. [Ese estereotipo] yo creo que nadie realmente lo cumple: (...) el estereotipo masculino de dominante, de agresivo, de insensible, de valiente, de fuerte, de todas estas cosas" (Julio, 21 años). Estas vivencias y perspectivas están adquiriendo cada vez más visibilidad pública gracias al trabajo de activismo y toma de la palabra protagonizado por los y las jóvenes trans, tanto en contextos presenciales como virtuales. En unos casos, participan en charlas y actividades escolares para dar a conocer sus experiencias y las discriminaciones sufridas. En otros casos, se involucran en el mundo asociativo o participan en vídeos y documentales distribuidos por las redes sociales o los medios de comunicación. Un buen número de varones trans, además, está tejiendo una amplia red de visibilidad por medio de sus videoblogs. Internet aparece aquí como una plataforma de investigación, comunicación y autorrepresentación con efectos positivos para estos jóvenes de cara a su visibilidad social y a la producción de imágenes no estigmatizantes de la realidad trans.

Por último, señalaremos que la irrupción de los "menores trans" y sus familias en los centros escolares está conllevando cambios hacia una mayor implicación de estas instituciones (y de las administraciones públicas) en el combate contra el acoso escolar y en la puesta en marcha de medidas, protocolos y planes de respeto a la diversidad. Los marcos explicativos que sustentan es- tas medidas institucionales no están exentos de contradicciones y combinan, en algunos casos, fundamentos coeducativos y críticos con la desigualdad con otros de carácter esencialista que refuerzan la cultura del rosa y del azul. En lo que respecta a la educación sexual, como veremos a continuación, esta sigue siendo percibida como deficitaria por parte de las personas entrevistadas.

\section{Los límites de la educación sexual recibida}

Nuestro marco legislativo establece que el sistema educativo debe contemplar la formación en salud sexual y reproductiva desde un enfoque no solo biofísico sino entendiendo que la sexualidad es parte del desarrollo integral de la personalidad y de la formación en valores, y reconociendo la diversidad de experiencias sexuales posibles (Arnau, 2016). En la práctica, sin embargo, la escasa educación sexual que se ofrece en los centros escolares permanece mayoritariamente centrada en las diferencias bio/fisiológicas y en las técnicas anticonceptivas o de prevención de infecciones de transmisión sexual, dejando en los márgenes las experiencias de las personas trans y de otras personas no normativas en cuanto a su orientación del deseo, sus capacidades o sus circunstancias corporales.

Es frecuente que esta formación en sexualidad (cuando se produce) se externalice por medio de talleres o sesiones puntuales dinamizadas por asociaciones y entidades especializadas sin que se incorpore a los centros como una cuestión educativa transversal. Como señaló Ander (20 años): “Aquí te daban una educación sexual que consistía en: vas a echar un polvo, ponte condón. Tienes la píldora del día después. Y un morreo también puede ser una relación sexual. Punto. (...) O sea, ya te digo que educación sexual la justita y muy hacia la heterosexualidad. (...) Si soy una tía y a mí me gustan las tías, de poco me sirve lo que hace un tío con una tía". Más allá de esas charlas, en el centro de Ander la sexualidad no se abordaba cotidianamente con el profesorado: "Tampoco es que hubiese un tabú, simplemente no salía el tema. Entonces, igual sí que había un tabú, no sé".

Como sugería Ander, los asuntos relacionados con la sexualidad, la afectividad o la identidad personal parecen estar excluidos de lo que resulta pensable y posible en el marco de la docencia reglada, produciéndose un acuerdo tácito entre profesorado y alumnado para dejarlos fuera del aula. No hay que perder de vista, además, que el profesorado carece de formación para abordar estas cuestiones (Pichardo y Moreno, 2015) y que uno de los principios sobre los que se asienta nuestro sistema escolar es la separación jerárquica entre lo cognitivo y lo afectivo, resultando en la devaluación de lo afectivo y en su desplazamiento a los espacios no formativos (Ross-Epp: 1999). El carácter externalizado y puntual (una vez al año o una vez en todo el ciclo escolar) de las charlas y talleres con las que se pretende cubrir la formación en sexualidad no hace sino poner de manifiesto esta jerarquía valorativa entre los aspectos cognitivos del aprendizaje -que son los importantes, los que ocupan el grueso de la actividad escolary los que tienen que ver con las prácticas afectivo-sexuales, que quedan relegados a los márgenes.

El aprendizaje de las sexualidades y los afectos, sin embargo, no está ausente en las escuelas; muy al contrario, se produce cada día de manera informal. En contraste con la desexualización del curriculum oficial, las relaciones afectivas y sexuales son compulsivamente discutidas y practicadas entre y en el interior de los grupos de pares (Mac An Ghaill, 1994: 90-91) bajo la influencia de unos modelos que vienen de fuera de la escuela: medios de comunicación, mundo virtual, industria audiovisual, música... y particularmente a través del consumo de pornografía, mayoritario entre los varones (Ballester et al., 2018). Es en estos espacios y en los del ocio juvenil (Ruiz-Repullo et al., 2020) o en las propias familias (De Stéfano, 2019), donde se construye el deseo, se reafirman o cuestionan los roles hegemónicos y donde, a menudo, se reproducen determinadas formas de violencia. 
En conclusión, las escasas acciones formativas en materia de sexualidad propuestas por los centros escolares han sido descritas por las personas entrevistadas como parciales e insuficientes. Incapaces, por tanto, de contrarrestar el aprendizaje informal de los modelos dominantes que se acaba de describir. Incluso cuando se ha incorporado en estas charlas la perspectiva de la diversidad, el espectro de posibilidades vitales y sexuales contemplado ha sido percibido como limitado. Así lo consideraba Víctor (20 años), a cuyo instituto acudió una asociación LGBT a impartir una charla sobre sexualidad y acoso escolar homofóbico en la que no se sintió reconocido porque la transexualidad no se nombró: "Se centraron un montón en la homosexualidad y en la prevención de embarazos no deseados. Ya está, es lo único que nos hablaron". Él lo vivió como una oportunidad perdida.

\section{Conclusiones}

A la vista de las experiencias recogidas en esta investigación, resulta evidente la necesidad de una educación sexual transversal con perspectiva de género que cuente con espacios formales para su desarrollo. Al mismo tiempo, con el fin de contrarrestar los mensajes, imaginarios y comportamientos estigmatizantes que continúan reproduciéndose entre la población joven, resultará imprescindible la inclusión en estos programas formativos de la diversidad de corporalidades, experiencias y sensibilidades emergentes en materia sexual y de género. Esta (co)educación sexual (atenta a las raíces de las violencias sexistas, homofóbicas y transfóbicas) necesitará poder desplegarse en entornos educativos en los que lo afectivo y lo cognitivo vayan de la mano, donde existan programas institucionalizados de ayuda mutua y acciones encaminadas a mejorar el clima socioemocional en un marco de diálogo, pensamiento crítico, convivencia respetuosa y cultura de paz (Del Barrio y van der Meulen, 2015: 113-114; Palomero y Fernández, 2001).

Trabajar pedagógicamente la capacidad para pensar de manera crítica y creativa el género, las sexualidades y los deseos aparece como uno de los retos que podrían revelarse más fructíferos. "Pensar de manera creativa implica ser conscientes de las posibilidades de transformar la realidad, del carácter procesual de esos cambios, de nuestra conciencia de que podemos hacer (y hacernos) las cosas de otra manera, de que podemos crear otros modelos" (López, 2004: 317). Sin duda, tenemos la oportunidad de fomentar guiones sexuales alternativos al patriarcal, cuyo eje no sean las relaciones de poder y de dominación, y de ampliar los imaginarios socioculturales sobre los afectos y sobre los cuerpos deseables y deseados, desbordando los estrechos márgenes de las dicotomías predominantes. Resulta esencial, además, trabajar críticamente sobre la masculinidad y explorar alternativas igualitarias con los varones. Si en este proceso se tienen en cuenta las experiencias innovadoras que ya están en marcha, si se legitiman las voces y vivencias de la juventud y se potencia su capacidad de reflexión crítica y creativa, los efectos beneficiosos sobre la salud sexual, las relaciones interpersonales y la autoestima corporal podrían ser importantes tanto para las personas trans (Galofre y Missé, 2015) como para todas las demás.

\section{Agradecimientos}

El presente trabajo se basa en una investigación financiada por el Programa de Formación del Profesorado Universitario FPU del Ministerio de Educación (España). Referencia de la ayuda: AP2009-3422.

\section{Referencias}

Araneta, A. (2013). Transfronteras: Un nuevo activismo mundial por la despatologización trans. En O. Moreno y L. Puche
(Eds.), Transexualidad, adolescencias y educación. Miradas multidisciplinares (pp. 89-109). Egales.

Arnau, S. (2016). La educación sexual y la diversidad funcional. TE. Trabajadores/as de la enseñanza, 356, 34-35.

Asociación Chrysallis Euskal Herria (2016). Chicos y chicas, identidad y cuerpo. Material didáctico. Chrysallis Euskal Herria y Red Loratuz. http://chrysallis.org.es/wp-content/uploads/2015/06/ material_didactico_v1.0.pdf

Ballarín, P. (2019). ¿Por qué llamamos educación a la que no coeduca? En R. Cobo (Ed.), La imaginación feminista. Debates y transformaciones disciplinares (pp. 165-200). La Catarata.

Ballester, L.; Orte, C. y Pozo, R. (2018). Nova pornografia i canvis en les relacions interpersonals. Balears front Espanya. Anuari de la Joventut de les Illes Balears, 2018, 231-265.

Barragán-Medero, F., y Pérez-Jorge, D. (2020). Combating homophobia, lesbophobia, biphobia and transphobia: A liberating and subversive educational alternative for desires. Heliyon, 6(10), e05225. https://doi.org/10.1016/j.heliyon.2020.e05225

Benítez, E. (Coord.) (2016). Ciberbullying LGBT-fóbico. Nuevas formas de intolerancia. COGAM.

Bozon, M. (2013). Sociologie de la sexualité. Armand Colin.

Butler, J. (2015) [1990]. El género en disputa. El feminismo y la subversión de la identidad. PUEG/Paidós.

Calvo, S. (2021). Educación sexual con enfoque de género en el currículo de la educación obligatoria en España: avances y situación actual. Educatio Siglo XXI, 39(1), 281-304. https://doi. org/10.6018/educatio.469281

Christensen, P. y Prout, A. (2002). Working with Ethical Symmetry in Social Research with Children. Childhood, 9(4): 477-497.

Coll Planas, G., Bustamante, G. y Missé, M. (2009). Transitant per les fronteres del gènere: Estratègies, trajectòries i aportacions de joves trans, lesbianes i gais. Secretaria de Joventut - Generalitat de Catalunya.

Connell, R.W. (1995). Masculinities. University of California Press.

De Stéfano, M. (2019). ¿De tal palo...? Parentalidad, género y violencia en la infancia de los hombres que han ejercido violencia contra sus parejas. Encrucijadas. Revista Crítica de Ciencias Sociales, 18, a1803. https://recyt.fecyt.es/index.php/encrucijadas/ article/view/79206.

Del Barrio, C. y Van der Meulen, K. (2016). Maltrato por abuso de poder entre iguales en el alumnado con discapacidad. Pensamiento psicológico, 14(1), 103-118.

Del Olmo, M. (2003). La construcción de la confianza en el trabajo de campo. Los límites de la entrevista dirigida. Revista de Dialectología y Tradiciones Populares, LVIII (1):191-220.

Elipe, P., Muñoz, M., y del Rey, R. (2017). Homophobic Bullying and Cyberbullying: Study of a Silenced Problem. Journal of Homosexuality, 65(5), 672-686. https://doi.org/10.1080/009183 69.2017.1333809

Feinberg, L. (2006 [1992]). Transgender Liberation: A Movement Whose Time Has Come. En S. Stryker y S. Whittle (Eds.), The Transgender Studies Reader (pp. 205-220). Routledge.

Gallardo, E. y Espinosa, M. (2021). Sobre la imposición del sistema género y la transfobia: Historias de niñxs crativxs con el género y jóvenes trans*. OBETS. Revista de Ciencias Sociales, 16(1), 115-134. https://doi.org/10.14198/OBETS2021.16.1.08

Galofre, P. y Missé, M. (2015). Introducción. En P. Galofre y M. Missé (Eds.), Políticas trans. Una antología de textos desde los estudios trans norteamericanos (pp. 19-29). Egales.

Hurtado, I. y Terrón, A. (2020). La educación sexual durante la Transición: modelando discursos y modulando voces. En J. Martínez y E. Perdiguero (Eds.). Genealogías de la reforma sanitaria en España (pp. 155-191). La Catarata.

Juliano, D. (2004). Excluidas y marginales. Una aproximación antropológica. Cátedra.

Juliano, D. (2017). Tomar la palabra. Mujeres, discursos y silencios. Bellaterra. 
León, C. M. y Aizpurúa, E. (2020). ¿Persisten las actitudes sexistas en los estudiantes universitarios? Un análisis de su prevalencia, predictores y diferencias de género. Educación XX1, 23(1), 275-296. https://doi.org/10.5944/educXX1.23629

López, M. (2004). Principios y argumentos de la Noviolencia. En B. Molina y F. A. Muñoz (Coords.), Manual de paz y conflictos (pp. 303-330). Universidad de Granada.

Mac An Ghaill, M. (1994). The Making of Men. Masculinities, Sexualities and Schooling. Open University Press.

Maquieira, V. (2005). Género, diferencia y desigualdad. En V. Maquieira y E. Beltrán (Eds.), Feminismos. Debates teóricos contemporáneos (pp. 127-190). Alianza.

Meyer, E. J. (2009). Gender, Bullying and Harassment. Strategies to End Sexism and Homophobia in Schools. Teachers College Press.

Missé, M. (2018). A la conquista del cuerpo equivocado. Egales.

Nieto, J. A. (2008). Transexualidad, intersexualidad y dualidad de género. Bellaterra.

Palomero, J. E. y Fernández, M. R. (2001). La violencia escolar: un punto de vista global. Revista Interuniversitaria de Formación del Profesorado, 41, 19-38.

Pibernat, M. (2021). Misoginia youtuber: conseguir audiencia con humor sexista. Investigaciones Feministas, 12(1), 47-56. https:// doi.org/10.5209/infe.69376

Pichardo, J. I. (Coord.) (2015). Abrazar la diversidad: propuestas para una educación libre de acoso homofóbico y transfóbico. Instituto de la Mujer y para la Igualdad de Oportunidades. NIPO.

Pichardo, J. I. y de Stéfano, M. (Eds.) (2015). Diversidad sexual y convivencia: Una oportunidad educativa. Universidad Complutense de Madrid.

Pichardo, J. I. y Moreno, O. (2015). Diversidad sexual y convivencia: perspectivas del profesorado. En J. I. Pichardo y M. de Stéfano (Eds.), Diversidad sexual y convivencia: Una oportunidad educativa (pp. 24-45). Universidad Complutense de Madrid.

Platero, R. (L.) (2014). Trans*exualidades. Acompañamiento, factores de salud y recursos educativos. Bellaterra.

Plummer, K. (2011). Foreword: Permanence and Change. Sexual Conduct-Thirty Years On. En J. Gagnon y W. Simon, Sexual Conduct. The Social Sources of Human Sexuality, Aldine.

Puche, L. (2018). Infancias y juventudes trans. Una aproximación desde la Antropología Social. Universidad Autónoma de Madrid.

Puche, L. (2021, en prensa). Itinerarios de conciliación corporal a través de la sexualidad y el placer. Un análisis de narrativas de jóvenes trans. Revista AIBR.
Chamberland, L. (2014). Violences homophobes, violences transphobes: le double jeu du genre au milieu scolaire. En K. Espinera, M. Thomas y A. Alessandrin (Eds.), Tableau noir: Les transidentités et l'école (pp. 77-94). L'Harmattan.

Ross-Epp, J. (1999). Escuelas, complicidad y fuentes de la violencia. En J. Ross-Epp, y A. Watkinson (Coords.), La violencia en el sistema educativo. Del daño que las escuelas causan a los niños (pp. 14-50). La Muralla.

Rubin, G. (1986). El tráfico de mujeres: notas sobre la "economía política" del sexo. Nueva Antropología, VIII(30), 95-145.

Ruiz-Repullo, C., López-Morales, J. y Sánchez-González, P. (2020). Violencia de género y abuso de alcohol en contextos recreativos. Revista española de drogodependencias, 45(2), 13-22. https:// www.aesed.com/upload/files/v45n2_1_carmenruiz-etal.pdf

Saleiro, S. P. (2017). Diversidade de género na infância e educação: contributos para uma escola sensível ao (trans)género. ex æquo, 36, 149-165. https://doi.org/10.22355/exaequo.2017.36.09

Sánchez, M., Penna, M. y De la Rosa, B. (2016). Somos como somos. Deconstruyendo y transformando la escuela. Los Libros de la Catarata.

Sanchís, R. (2021). Els nostres cossos, els nostres drets (Guia d'Educació Sexual). Generalitat Valenciana.

Serrano, J. (2007). Whipping Girl. A Transsexual Woman on Sexism and the Scapegoating of Femininity. Seal Press.

Stryker, S. (2008). Transgender History. Seal Press.

Suess, A. (2018). Derechos de las personas trans e intersex revisión del marco legislativo en el contexto español desde una perspectiva de despatologización y derechos humanos. DS: Derecho y salud, 28 (Extra 1), 97-115.

Takács, J. (2006). Social Exclusion of Young Lesbian, Gay, Bisexual and Transgender People (LGBT) in Europe. ILGA Europe.

Travers, A. (2014). Transformative Gender Justice as a Framework for Normalizing Gender Variance among Children and Youth. En E. Meyer y A Pullen-Sansfaçon (Eds.), Supporting Transgender $\mathcal{E}$ Gender Creative Youth. Schools, Families and Communities in Action (pp. 54-68). Peter Lang.

Trinidad, A., Carrero, V. y Soriano, R. (2006). Teoría fundamentada "Grounded Theory". La construcción de la teoría a través del análisis interpretacional. CIS. 